\title{
Acute stent thrombosis after off-pump coronary bypass surgery: A new and avoidable complication?
}

\author{
J. M. Alvarez, FRACS, ${ }^{a}$ L. Chen, MB, BS, ${ }^{a}$ and I. N. Sinclair, FRACP, ${ }^{b}$ Perth, West Australia
}

1 majority of percutaneous revascularization procedures incorporate percutaneous transluminal coronary artery stenting with balloon percutaneous transluminal coronary angioplasty (PTCA). Acute coronary stent thrombosis, however, remains an uncommon yet serious complication. ${ }^{1}$ Recently, off-pump coronary artery bypass (OPCAB) surgery has gained widespread popularity. ${ }^{2}$ However, the effect of OPCAB surgery on coagulation-fibrinolytic homeostasis is unclear. We report 2 cases of acute coronary stent thrombosis in previously stented ungrafted coronary arteries (ie, angiographically normal) after OPCAB surgery. Both patients had normal coagulation profiles.

\section{Clinical Summaries}

PATIENT 1. A 42-year-old male smoker was admitted with unstable angina. The patient's sole previous history was of successful primary right coronary artery (RCA) PTCA after an inferior acute myocardial infarction (AMI) in 1995. Despite treatment with aspirin and intravenous heparin and glyceryl trinitrate, angina persisted; cardiac catheterization revealed a 90\% discrete $(19 \mathrm{~mm})$ RCA stenosis and a total left anterior descending coronary artery (LAD) occlusion, and left ventricular function was normal. A $35 \times$ 8 -mm Tetra stent was successfully deployed to the RCA, and daily clopidogrel was added (Figure 1, $A$ and $B$ ).

Persistent angina with demonstrable reversible anterior wall ischemia on thallium scanning resulted in OPCAB surgery 5 days later. Aspirin and clopidogrel were ceased 4 days beforehand. A pedicled left internal thoracic artery was grafted to the LAD with the Octopus III stabilizer (Medtronics). Only aspirin was recommenced 24 hours later. Recovery was uneventful for 72 hours, when unheralded chest pain associated with inferior ST-segment elevation and hemodynamic collapse occurred. Immediate cardiac catheterization revealed a normally functioning left internal thoracic artery graft but acute RCA stent thrombosis (Figure 1, C). This was successfully opened by means of PTCA, and ReoPro (Eli Lilly) was commenced (Figure 1,D). Despite a perioperative AMI

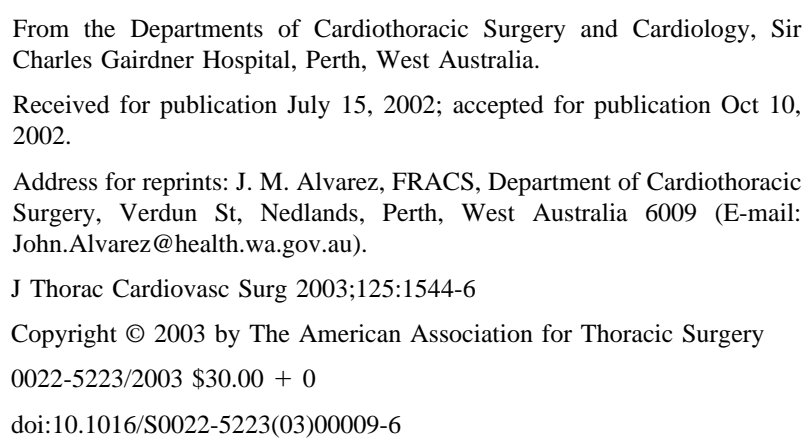

(creatine kinase, $2900 \mathrm{U} / \mathrm{L}$ ), uneventful discharge home occurred 19 days later.

PATIENT 2. A 61-year-old man admitted with unstable angina was treated with aspirin and intravenous heparin and glyceryl trinitrate. Six months previously, 4 stents were successfully and electively deployed to the LAD, RCA, circumflex artery, and diagonal artery. Risk factors were hypertension and hyperlipidemia. Angiography revealed RCA and circumflex artery in-stent restenosis $(\geq 95 \%)$, the LAD stent was pristine (Figure 2, $A$ ), and left ventricular function was normal. Ongoing angina resulted in OPCAB surgery 4 days later. Saphenous vein grafts were performed to the circumflex artery and RCA by using the Octopus III stabilizer. Aspirin was restarted within 24 hours, and postoperative recovery was uneventful.

Seven days later, unheralded acute pulmonary edema and ventricular fibrillation occurred. After cardiopulmonary resuscitation, immediate angiography revealed patent saphenous vein grafts and a $100 \%$ LAD stent thrombosis (Figure 2, B), which was successfully treated with PTCA and ReoPro (Figure 2, $C$ ). Recovery from the AMI (creatine kinase, $3800 \mathrm{U} / \mathrm{L}$ ) was complicated by deep sternal infection successfully managed with a bilateral myocutaneous pectoralis flap with an omental graft. The patient was discharged home 31 days after OPCAB surgery.

\section{Discussion}

We report 2 cases of acute coronary stent thromboses after OP$\mathrm{CAB}$ surgery. In both cases the thrombosed stents exhibited no evidence of in-stent pathology preoperatively, and thus the respective coronary arteries were ungrafted. Direct surgical trauma to coronary stents causing stent thrombosis has been reported with coronary artery bypass grating $(\mathrm{CABG})$ with cardiopulmonary bypass (CPB). ${ }^{3}$ Ostensibly, such trauma could occur with cardiac manipulation during OPCAB surgery. However, the coronary anatomy in both cases precluded the need for such manipulation to infringe on these stents.

Aspirin is traditionally recommenced within 24 hours after CABG. Approximately $10 \%$ of all patients with CABG in our unit (400 patients per year) over the last 5 years have had previous percutaneous transluminal coronary artery stenting, with many of these stented coronaries being pristine, thus not requiring coronary grafting. The vast majority $(>95 \%)$ of these operations were performed with $\mathrm{CPB}$, and acute stent thrombosis never occurred. Ostensibly, the anticoagulant effect of CPB and postoperative aspirin prevents acute stent thrombosis in these angiographically pristine vessels.

Despite initial enthusiasm, the purported benefits from OPCAB surgery remain unproven. ${ }^{2}$ With OPCAB surgery, the optimal heparin dose (we used $1 \mathrm{mg} / \mathrm{kg}$ ) and whether to reverse this effect with protamine and at what dose (we used 100 and $50 \mathrm{mg}$, respectively, for patients 1 and 2) is unclear. However, this is at least one third of the heparin-protamine requirements with $\mathrm{CPB}$. 

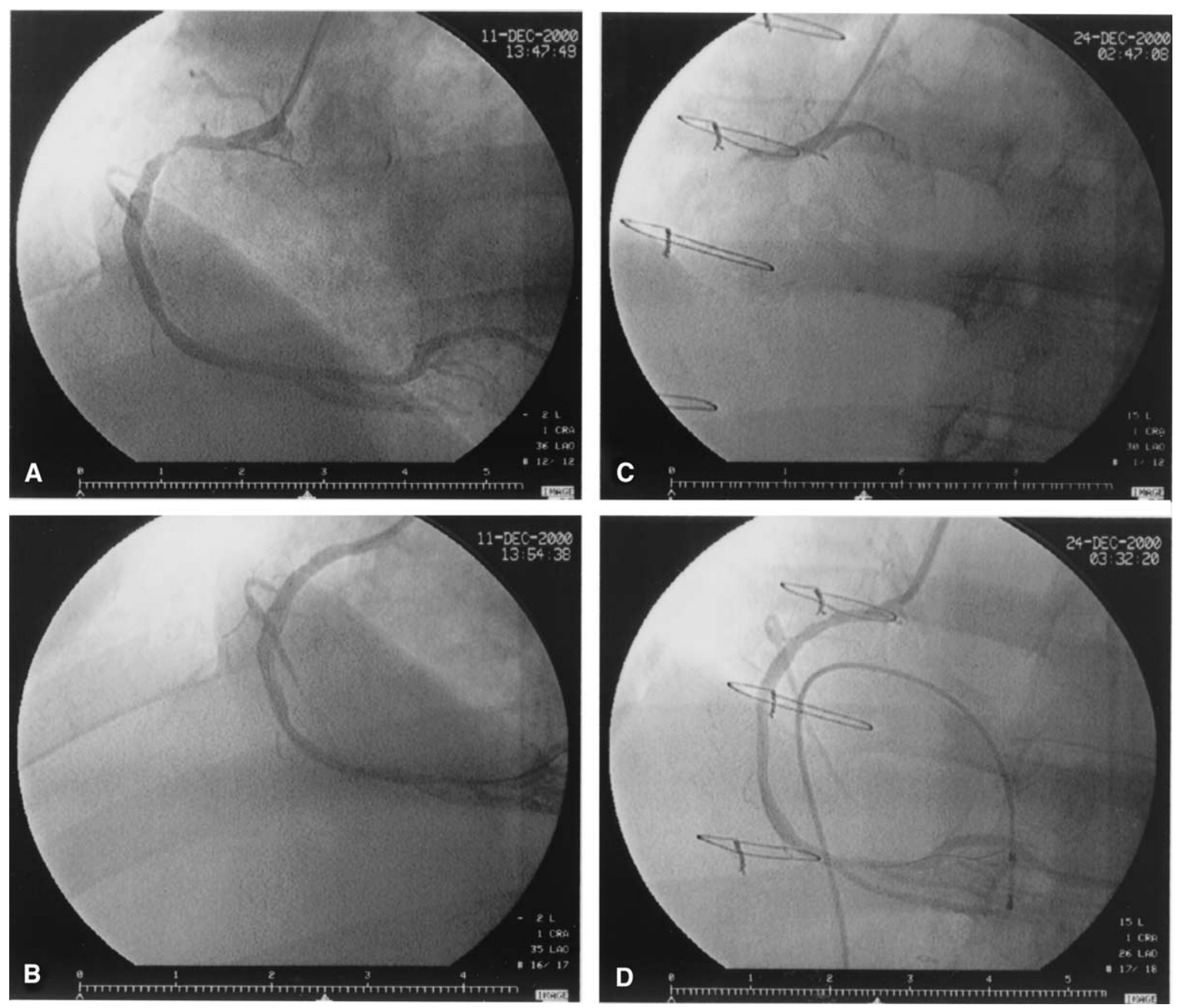

Figure 1. Left anterior oblique angiogram of the RCA. Before OPCAB surgery: $A$, severe proximal RCA stenosis; $B$, after stenting, with excellent luminal diameter. After OPCAB surgery: $C$, acute in-stent thrombosis; $D$, after emergency RCA angioplasty and ReoPro.
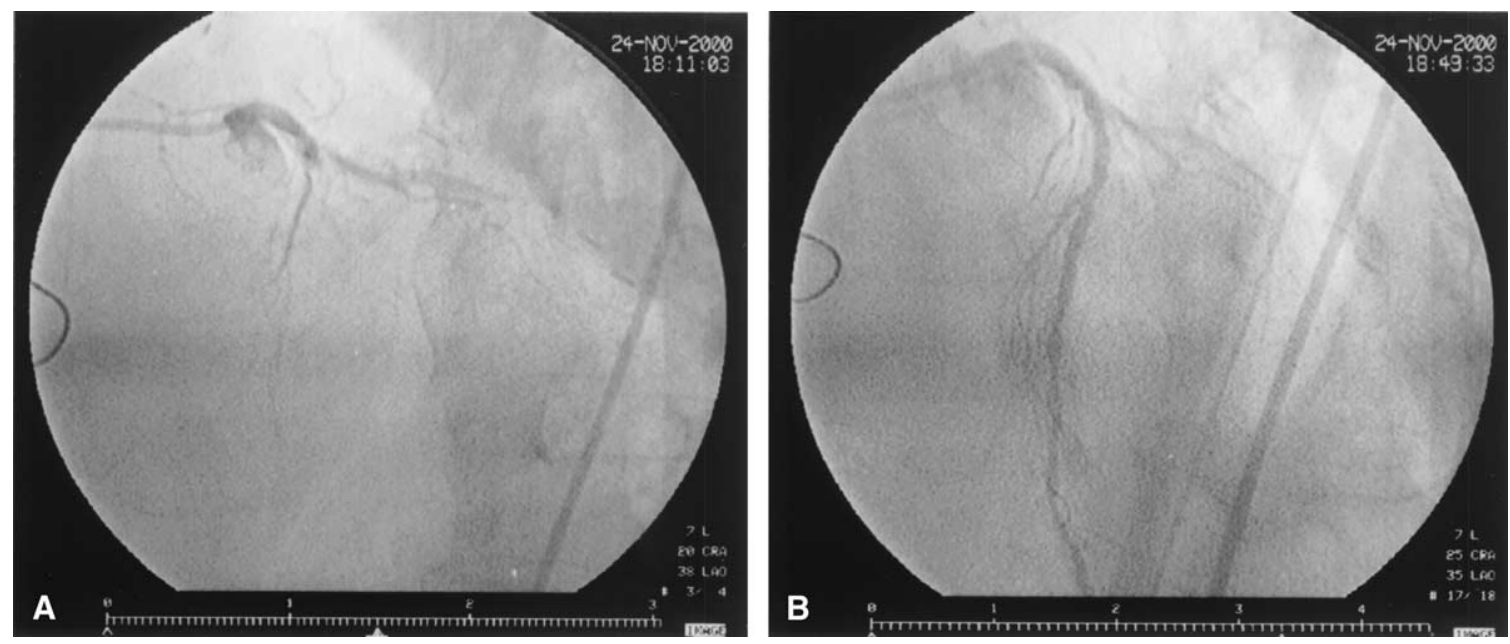

Figure 2. Post-OPCAB left anterior oblique angiogram of the LAD: $A$, acute LAD stent thrombosis; $B$, result after emergency LAD angioplasty and ReoPro. 
Furthermore, all major operations induce a prothrombotic diathesis. With OPCAB surgery, unlike $\mathrm{CABG}$ with $\mathrm{CPB}$, platelet numbers and function remain preserved. ${ }^{4}$ Also, a prothrombotic effect partly attributed to increased platelet reactivity has been demonstrated after OPCAB surgery. ${ }^{4}$

We have performed OPCAB surgery in 150 patients without evidence of acute coronary thrombosis. These 2 patients were the first to have OPCAB surgery with patent stents. In these patients with stented yet pristine coronary arteries, sole reliance on aspirin seems imprudent. We thus add clopidogrel. With this strategy, a further 8 patients with patent stents had uncomplicated OPCAB surgery.

\section{References}

1. Cutlip DE, Baim DS, Ho KK, Popma JJ, Lansky AJ, Cole DJ, et al. Stent thrombosis in the modern era: a pooled analysis of multicenter coronary stent clinical trials. Circulation. 2001;103:1967-71.

2. Yacoub M. Off pump coronary bypass surgery. In search of an identity. Circulation. 2001;104:1743-5.

3. Tovar EA, Borsari A. Effects of surgical manipulation on coronary stents: should surgical strategy be altered? Ann Thorac Surg. 1997;63: $37-40$.

4. Valleley MP, Bannon PG, Kritharidis L. The systemic inflammatory response syndrome and off-pump cardiac surgery. Heart Surg Forum. 2001;4(suppl I):S7-13.

\title{
Proximal prolapse of aortic intimal flap: A rare complication of acute type $A$ aortic dissection
}

\author{
G. Hossein Almassi, MD, Milwaukee, Wis
}

\section{A}

cute type A aortic dissection requires emergency surgical repair to prevent the known life-threatening complications of rupture, cardiac tamponade, acute coronary ischemia, and branched arterial occlusion. Prolapse of the intimal flap into the distal ascending aorta and the aortic arch has been reported in cases of circumferential tear of the aortic intima. ${ }^{1-4}$ We report a case of acute type A aortic dissection with intussusception of the intimal flap proximally into the aortic root and the left ventricular outflow tract, causing global myocardial ischemia.

A 75-year-old man had acute chest pain and shortness of breath. He was hypotensive, and the electrocardiogram disclosed global ST-segment depression in leads I, II, III, AVL, and AVF and precordial leads $\mathrm{V}_{2}$ to $\mathrm{V}_{6}$. Medical history included severe chronic renal insufficiency, coronary artery bypass grafting 10 years earlier, and repair of abdominal aortic aneurysm on 2 occasions 30 years and 7 years before the current presentation. A noncontrast computed tomographic (CT) scan of the chest and abdomen revealed extensive aneurysmal disease of the ascending and descending thoracic aorta with possible dissection of the descending and the remaining abdominal aorta. The patient was transferred to the cardiac catheterization laboratory, where multi-

\footnotetext{
From the Division of Cardiothoracic Surgery, Medical College of Wisconsin, Milwaukee, Wis.

Received for publication July 30, 2001; accepted for publication Feb 15, 2002.

Address for reprints: G. Hossein Almassi, MD, Division of Cardiothoracic Surgery, Medical College of Wisconsin, Milwaukee, Froedtert East Clinic, PO Box 26099, Milwaukee, WI 53226 (E-mail: galmassi@mcw.edu).

J Thorac Cardiovasc Surg 2003;125:1546-8

Copyright (C) 2003 by The American Association for Thoracic Surgery $0022-5223 / 2003 \$ 30.00+0$

doi:10.1016/S0022-5223(02)73593-9
}

ple attempts at cannulation of the coronary ostia were unsuccessful. An aortic angiogram was inconclusive for dissection of the ascending aorta and failed to show the coronary arteries. Transesophageal echocardiography revealed an intimal flap in the root of the aorta close to the aortic valve prolapsing into the left ventricle in diastole (Figure 1). The aortic valve appeared intact. The patient was transferred to the operating room in cardiogenic shock and receiving high-dose inotropic support.

Operative findings included a circumferential tear of the aortic intima in the midascending aorta at the level of old saphenous vein grafts. The intimal flap had totally prolapsed into the left ventricular outflow tract, covering the ostia of the left and right coronary arteries. During deep hypothermic circulatory arrest and retrograde cerebral perfusion, the dissection was repaired with an aortic valve conduit. Coronary artery bypass grafting was also performed.

Although infrequent, circumferential intimal tear of the ascending aorta can lead to intussusception of the intimal flap distally into the aortic arch in line with the direction of blood flow. ${ }^{1-4}$ Proximal prolapse into the aortic root and left ventricular outflow tract is very unusual. The presentation in this patient might have been due to the presence of a saphenous vein graft suture line in the ascending aorta, keeping the distal flap from prolapsing, and to an extremely low cardiac output, despite high-dose inotropic support. Aortography was not diagnostic for ascending aortic dissection because the intimal flap had intussuscepted into the left ventricular outflow tract, and therefore there was absence of a false channel in the ascending aorta. We did not find any prior report on this complication in the English literature.

Contrast-enhanced spiral CT scanning of the chest is a fast, noninvasive, and sensitive test for the diagnosis of acute aortic dissection. ${ }^{5}$ Contrast CT scan was not obtained in this patient because of severe renal insufficiency. The diagnosis was ultimately confirmed by means of transesophageal echocardiography. Transesophageal echocardiography can accurately establish the diagnosis of ascending aortic dissection by demonstrating a mobile flap or 